\title{
Use of microneedle device to enhance dermal absorption: study on ex vivo human skin
}

\begin{abstract}
The present study investigated the effects of microneedle roller device on dermal delivery of Rhodamine 123 on ex vivo human skin. The effect of microneedle treatment on transepidermal water loss (TEWL) was also evaluated. Permeation studies of Rhodamine 123 through full-thickness human skin were conducted using modified Franz diffusion cells. The results showed that TEWL increased with the number of passes of the roller device. In addition, results of the in vitro permeation studies revealed marked increase of the absorption and the distribution of Rhodamine 123 through microneedle-treated skin. The increase of skin absorption was dependent on needle length. Thus, microneedle roller device markedly increased the absorption and distribution of Rhodamine 123 in ex vivo human skin and could be suitable for clinical and preclinical use in order to enhance dermal delivery of test molecules.
\end{abstract}

Volume 2 Issue I - 2018

\author{
Hanan Osman Ponchet, Marion Alriquet, \\ Magali Kouidhi, Karine Sevin, Alexandre \\ Gaborit, Claire EWilson \\ Nestlé Skin Health-Galderma, Sophia Antipolis, France
}

\begin{abstract}
Correspondence: Hanan Osman-Ponchet PhD, Nestlé Skin Health R\&D, Les Templiers, 2400 Route des Colles, BP87, F-06902, Sophia Antipolis, France, Tel +33(0)492386786, Fax +33(0)493957070, Email hanan.osman-ponchet@galderma.com
\end{abstract}

Received: October 02, 2017 | Published: January 24, 2018

Keywords: ex vivo, stratum corneum, microneedle, cryomatrix, haematoxylin

Abbrevations: SC, stratum corneum; MN, microneedle; TEWL, transepidermal water loss

\section{Introduction}

Skin constitutes an excellent barrier for the transdermal delivery of hydrophilic or high molecular weight drugs. Low efficacy demonstrated in clinical trials for some topical drugs is mainly due to low skin penetration ability related to the efficient barrier properties of stratum corneum (SC). Transdermal delivery of molecules in the skin is limited by the full epidermis, not just stratum corneum. ${ }^{1}$ In order to overcome the skin barrier, different chemical and physical methodologies have been investigated to enhance skin penetration. Among the physical methods, one presents the perforation of the skin with microneedles $(\mathrm{MN})$ that disrupts the barrier of the skin and creates pores inducing an increase of penetration. Microneedle technology offers an efficient and minimally invasive strategy to enhance transdermal drug delivery compared to conventional transdermal patches and intravenous injections..$^{2-6}$

There are several models of MN devices differing by the length and the number of needles. The length of needles varies between $0.2 \mathrm{~mm}$ and $2.5 \mathrm{~mm}$. Needles longer than $0.2 \mathrm{~mm}$ may cause pain and require a pre-anaesthetization of the skin. Depending on the needle's length, MN devices may be used for a specific application such as transdermal delivery, collagen induction therapy, hyper pigmentation, anti-wrinkles, hair regeneration, regrowth, cellulite treatment and scar treatment. There are many reports in the literature describing the use of MN devices in clinical situation. ${ }^{7}$ However, to our knowledge; nothing is described about the use of $\mathrm{MN}$ device in ex vivo human skin, especially with regard to transdermal delivery in vitro. The aim of this work was to validate the use of commercially available microneedle roller device with different needle lengths on ex vivo human skin to improve dermal delivery of test molecules.

\section{Materials and methods}

Four different models of microneedles roller device with different needle lengths $(0.2,0.5,1$ and $1.5 \mathrm{~mm})$ were used through the study.
Fresh human skin samples (full-thickness) from three different donors were used. Skin samples were all taken from abdominal region. The integrity of each skin sample was assessed by measuring transepidermal water loss (TEWL) with a Tewameter ${ }^{\circledR}$ TM300 (Courage+Khazaka electronic GmbH, Germany) measuring device. The acceptance criterion for TEWL values for intact non rolled skin was below $9 \mathrm{~g} / \mathrm{m}^{2} / \mathrm{h}$.

Skin samples were rolled with microneedles roller device according to manufacturer's instruction (Figure 1). After rolling, skin samples were placed in Transwell ${ }^{\circledR}$ inserts in 6-well plate prefilled with $2 \mathrm{~mL}$ of long term skin culture medium (Biopredic, France). In order to define the surface of treatment area, a $1 \mathrm{~cm}^{2}$ glass cylinder was glued on the top of the skin samples (Figure 2).

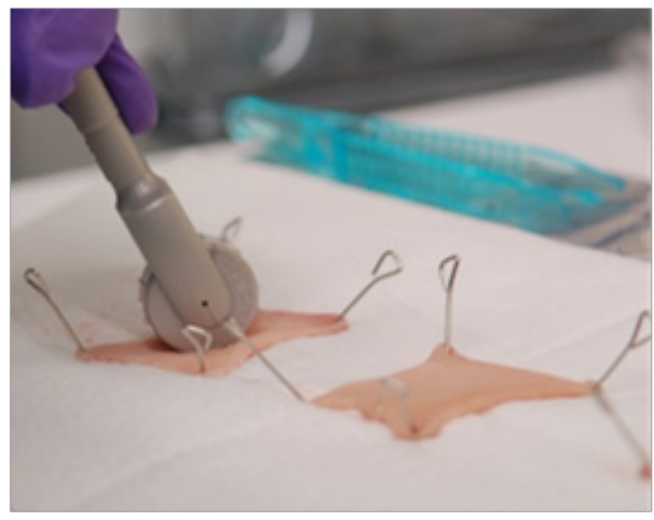

Figure 1 Rolling of ex vivo human skin sample using a micro needle roller device

A solution of Rhodamine 123(1\% in DMSO) was applied on skin surface at amount of $100 \mathrm{mg} / \mathrm{cm}^{2}$. Skin samples were then maintained in cell incubator set at $37^{\circ} \mathrm{C}, 5 \% \mathrm{CO}_{2}$ and saturated hygrometry under gentle shaking for 6hours. Each condition was performed in triplicate. Non rolled skin samples were used as controls.

Concentrations of Rhodamine 123 in receptor fluid were measured by spectrofluorometry (excitation wavelength $485 \mathrm{~nm}$ and emission 
wavelength $590 \mathrm{~nm}$ ) against a calibration curve ranged between $0.01 \mu \mathrm{M}$ and $10 \mu \mathrm{M}$.

Skin biopsy samples were embedded in Cryomatrix ${ }^{\mathrm{TM}}$ embedding resin (Thermo Scientific, USA). Cross sections $(6 \mu \mathrm{m})$ were cut using a cryomicrotome (Cryotome FSE, Thermo Electron, USA). Skin sections were mounted on glass microscope slides (SuperFrost ${ }^{\circledR}$ Plus, Thermo Scientific, USA) and stained using haematoxylin. Skin sections were observed using a Nikon Eclipse 80i microscope equipped with a DS-Ri1 high resolution camera (Nikon Instruments Inc., USA). Distribution of Rhodamine 123 in skin sections was visualized by epi-fluorescence using a TRITC fluorescence filter cube. Fluorescence related to Rhodamine 123 was analyzed using Image J software.

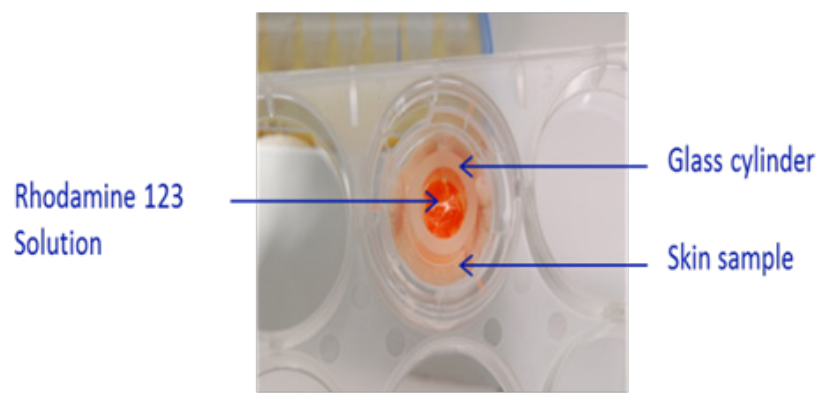

Figure 2 Ex vivo skin sample mounted on Transwell ${ }^{\circledR}$ insert with defined treatment surface area using a glass cylinder.

\section{Results and discussion}

\section{Effect of microneedles device on TEWL}

Transepidermal water loss measurements were taken to study disruption of skin barrier. Rolling of ex vivo human skin with microneedles device markedly increased the transepidermal water loss (Figure 3). Increase of TEWL was maximal after 10 passes of microneedles device and became stable after 20 passes of. This is due to the formation of microholes or microchannels and indicates increasing disruption of the skin barrier. The optimal use of microneedles device in our conditions is set between 10 and 20 passes. Our results are in accordance with previous results showing that TEWL increased as a function of the number of passes on rat skin. ${ }^{8}$

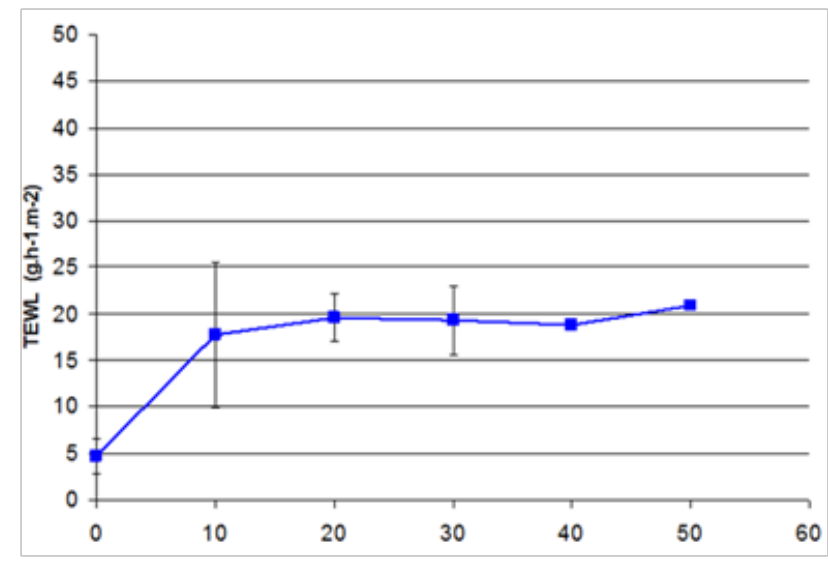

Number of passes of micro needle device.

Figure 3 Effect of micro needle device on TEWL on ex vivo human skin.

\section{Effect of microneedles device on dermal absorption of Rhodamine I 23}

\section{Skin absorption}

The result presented in Figure 4 indicates that the use of microneedles device markedly increased the skin absorption of Rhodamine 123 in the receptor fluid in ex vivo human skin. Treatment with increasing lengths of microneedles resulted in increased absorption of Rhodamine 123 indicating increased the degree of disruption of the skin barrier. Skin absorption increase ranged from 2 -folds (needle length $0.5 \mathrm{~mm}$ ) to 7 -folds (needle length $1.5 \mathrm{~mm}$ ). Our results are in accordance with previous results showing that treatment with increasing lengths of microneedles resulted in increased TEWL. ${ }^{9}$

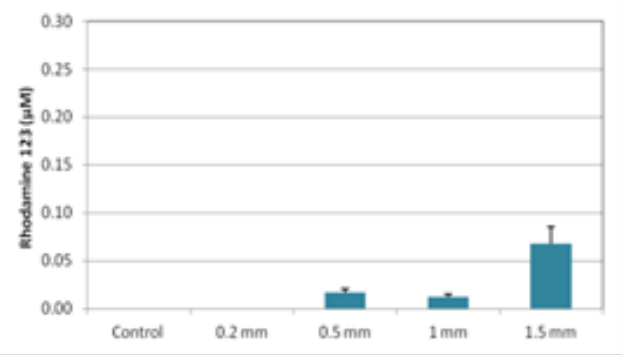

Figure 4 Effect of needle length on dermal absorption of Rhodamine 123 (Donor H2).

\section{Skin penetration}

The results presented in Figure 5 indicate that the use of microneedles device markedly increased the penetration of Rhodamine 123 in ex vivo human skin. Indeed, bright high intensity fluorescence was clearly visible in upper layers of skin mainly around the micro channels created by the microneedles, indicating the diffusion of the fluorescent molecule through the conduits created. A wide area of fluorescence could be observed deeper in the skin treated with needles longer than $0.2 \mathrm{~mm}$. In addition, fluorescence increased with increasing lengths of microneedles indicating that penetration of Rhodamine 123 in the skin increased with increasing lengths of microneedles. On the other hand, the micro channels created by the different needle models were clearly visible on skin sections (Figure 6).
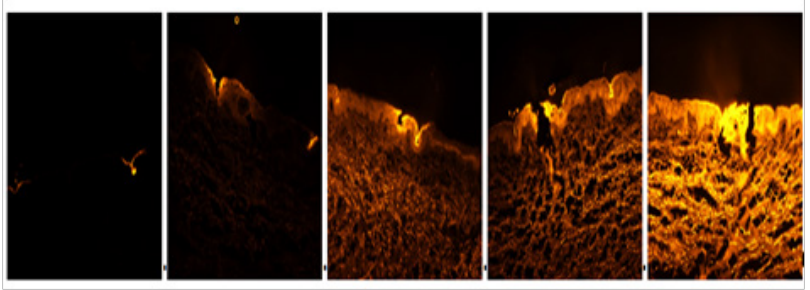

Needles' length 0mm, $1.5 \mathrm{~mm}, 0.2 \mathrm{~mm}, 0.5 \mathrm{~mm}, 1 \mathrm{~mm}$.

Figure 5 Fluorescence microscopy analysis: evaluation of penetration of Rhodamine 123 as function of needle length in ex vivo human skin (donor $\mathrm{H} 2$, $\mathrm{x} 4$ magnification, exposure time $1 / 15 \mathrm{eme}$ second).

Image analysis (Figure 7) shows that fluorescence increased with increasing lengths of microneedles indicating that penetration of Rhodamine 123 in the skin increased with increasing lengths of microneedles. Increase of Rhodamine 123 in the skin induced by needle length of $0.2 \mathrm{~mm}$ ranged from 2 -folds (donor H2) to 12 -folds (donor $\mathrm{H} 1$ ), and that induced by needle length of $1.5 \mathrm{~mm}$ ranged between 
12-folds (donor H1) and 19-folds (donor H2). It should be noted that in donor $\mathrm{H1}$, a 32 -fold increase was induced by needle length of $1 \mathrm{~mm}$. One would expect a continuous increase as observed in donor $\mathrm{H} 2$, the decrease of fluorescence from $1 \mathrm{~mm}$ to $1.5 \mathrm{~mm}$ observed in donor $\mathrm{H} 1$ may be related to the skin sample itself. Overall, the results clearly indicate a marked increase of dermal penetration of Rhodamine 123 by microneedles; despite a high inter individual variability.

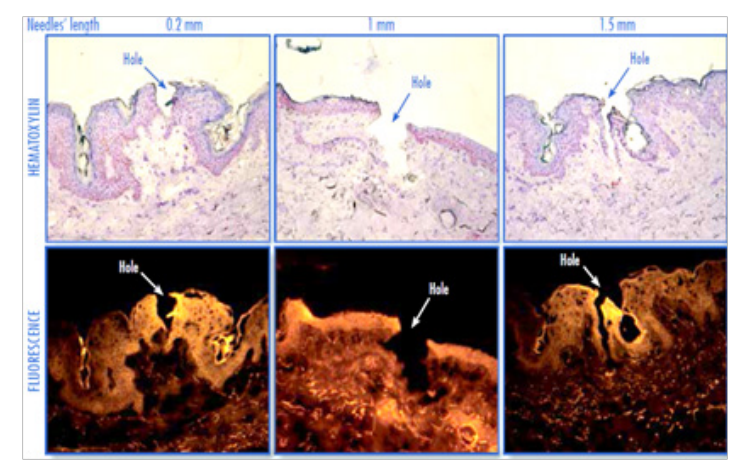

Figure 6 Visualization of micro channels created by micro needle roller devices in ex vivo human skin. Upper panel: histological analysis by optical microscopy (haematoxylin stain). Lower panel: fluorescence microscopy (donor H1, x20 magnification).
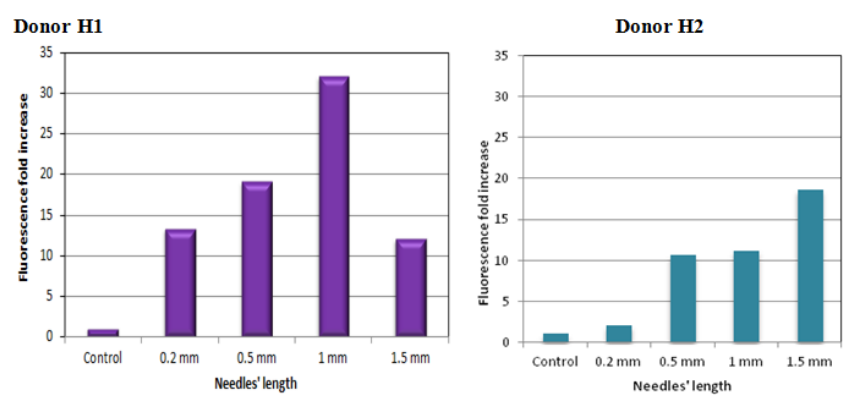

Figure 7 Effect of needle length on skin distribution of Rhodamine 123 in ex vivo human skin from donors $\mathrm{H} 1$ and $\mathrm{H} 2$.

Regarding the needle length, a good correlation was shown between skin absorption and skin distribution of Rhodamine 123 (Figure 8).

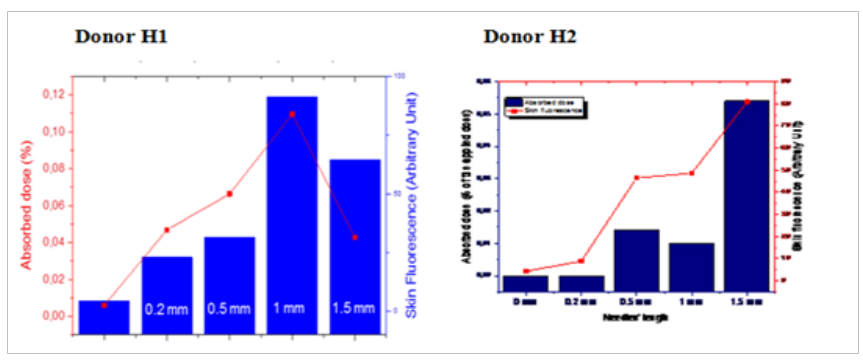

Needles' length: $0 \mathrm{~mm}, 0.2 \mathrm{~mm}, 0.5 \mathrm{~mm}, 1 \mathrm{~mm}, 1.5 \mathrm{~mm}$.

Figure 8 Correlation between skin absorption and skin distribution of Rhodamine 123.

\section{Conclusion}

The results of this work clearly showed that the use of microneedles roller device markedly increased the absorption and the distribution of Rhodamine 123 on ex vivo human skin. The increase of was dependent on needle's length. In addition, the use of the smallest needle of $0.2 \mathrm{~mm}$ was shown to considerably increase dermal absorption of Rhodamine 123 in ex vivo human skin samples. This model may be easily used in clinical situation as no anesthetic treatment is needed prior to use this device.

\section{Acknowledgements}

None.

\section{Conflicts of interest}

The authors declared that there are no conflicts of interest.

\section{References}

1. Andrews SN, Jeong E, Prausnitz MR. Transdermal delivery of molecules is limited by full epidermis, not just stratum corneum. Pharm Res. 2013;30(4):1099-1109.

2. Nguyen HX, Banga AK. Enhanced skin delivery of vismodegib by microneedle treatment. Drug Deliv Transl Res. 2015;5(4):407-423.

3. Kim YC, Park JH, Prausnitz MR. Microneedles for drug and vaccine delivery. Adv Drug Deliv Rev. 2012;64(14):1547-1568.

4. Prausnitz MR. Microneedles for transdermal drug delivery. Adv Drug Deliv Rev. 2004;56(5):581-587.

5. Stoeber B, Liepmann D. Arrays of hollow out-of-plane microneedles for drug delivery. J Microelectromech Syst. 2005;14(3):472-479.

6. Tuan-Mahmood TM, McCrudden MT, Torrisi BM, et al. Microneedles for intradermal and transdermal drug delivery. Eur $J$ Pharm Sci. 2013;50(5):623-637.

7. Ita K. Transdermal delivery of drugs with microneedles-potential and challenges. Pharmaceutics. 2005;7(3):90-105.

8. Kalluri H, Kolli CS, Banga AK. Characterization of microchannels created by metal microneedles: formation and closure. AAPS J. 2011;13(3):473481 .

9. Badran MM, Kuntsche J, Fahr A. Skin penetration enhancement by a microneedle device (Dermaroller) in vitro: dependency on needle size and applied formulation. Eur J Pharm Sci. 2009;36(4-5):511-523. 